Practice Lesson of the Week

\title{
Treatment for lymph node tuberculosis
}

BMJ 2010; 340 doi: http://dx.doi.org/10.1136/bmj.c63 (Published 10 March 2010) Cite this as: BMJ 2010;340:c63

- Article

- Related content

- Metrics

- Responses

- Peer review

-

Rob S Sellar, registrar in haematology 1 , E L Corbett, reader in infectious and tropical diseases 2 , Shirley D'Sa, consultant haematologist ${ }^{13}$, David C Linch, professor of haematology and head of department ${ }^{14}$, Kirit M Ardeshna, consultant haematologist 13

\section{Author affiliations}

Correspondence to: K M Ardeshna kirit.ardeshna@uclh.nhs.uk

Accepted 9 November 2009

Treatment for lymph node tuberculosis must be preceded by adequate pathological investigation of the lymphadenopathy

Among the numerous causes of lymphadenopathy, tuberculosis and lymphoma are both relatively common and potentially curable. The clinical features of tuberculous lymphadenitis overlap with those of lymphoma: some patients with each condition are asymptomatic apart from painless swelling, whereas others are unwell and have systemic symptoms such as fevers, weight loss, or night sweats. Accurate diagnosis depends on confirmation with appropriate pathology tests. We present three patients with lymphoma who were initially misdiagnosed as having tuberculosis. In each case this led to a delay in the treatment of the lymphoma, and in one case, chronic side effects from the unnecessary exposure to tuberculosis treatment compromised the treatment of the lymphoma. Lymph node biopsy and adequate histopathological assessment would have prevented these misdiagnoses and should be mandatory in suspected cases of lymph node tuberculosis when microscopy and cytology of fine needle aspirate does not show alcohol and acid fast bacilli, caseating granuloma, or granuloma with Langerhan's cells.

\section{Case 1}

A 24 year old man was referred to a respiratory clinic with a four month history of lethargy, weight loss, and night sweats, together with a mass on the right side of his neck and a prominent right tonsil. More recently he had developed a cough and had had three episodes of green sputum. He was previously well and had no personal or family history of tuberculosis. He was born in the United Kingdom to Pakistani parents and received a BCG vaccination at school. He had visited Pakistan two years previously, and one year before presentation had come into contact with tuberculosis through his work.

Examination found a large neck mass $(8 \mathrm{~cm}$ by $5 \mathrm{~cm}$ ) but was otherwise unremarkable. A chest $x$ ray three months before had been normal. Computed tomography of the neck and thorax showed a nodal mass involving the cervical lymph nodes and extending to the supraclavicular region. There was no 
mediastinal lymphadenopathy. Full blood count and renal and liver function tests were unremarkable. Inflammatory markers were raised. Fine needle aspirate cytology was reported as showing granulomatous lymphadenitis. The patient was diagnosed with lymph node tuberculosis and given Rifater (rifampicin, isoniazid, and pyrazinamide) and ethambutol. Two months later the lump was unchanged; cultures for Mycobacterium tuberculosis were negative. Treatment was modified to isoniazid, ciprofloxacin, ethambutol, and prednisolone because of nausea and abnormal liver function tests. Ethambutol was subsequently discontinued because colour blindness developed as an adverse effect of the drug.

Six months later the lump was unchanged and he was systemically unwell with fever, weight loss, and night sweats. An ultrasound scan revealed a solid lump, from which no pus could be aspirated. Computed tomography showed a necrotic, right sided neck mass together with subcarinal and axillary lymphadenopathy and splenomegaly. An excision lymph node biopsy performed seven months after his presentation confirmed nodular sclerosing Hodgkin's lymphoma. He arrived at the lymphoma clinic in a wheelchair because of peripheral neuropathy induced by isoniazid. He was given escalated BEACOPP (bleomycin, etoposide, doxorubicin, cyclophosphamide, vincristine, procarbazine, and prednisolone) chemotherapy but vincristine, a cornerstone of treatment, was omitted because of its potential to worsen his neuropathy.

\section{Case 2}

A 25 year old Brazilian woman presented with right sided cervical lymphadenopathy four months after arriving in Britain. She had no other symptoms and the rest of her examination was normal. Routine blood tests, including a full blood count, were normal, as was her chest x ray. An ultrasound scan of right neck revealed multiple $1 \mathrm{~cm}$ lymph nodes that had become confluent. Fine needle aspirate cytology showed lymphocytes at different stages of maturation and comment was made that no malignant cells were seen. No comment was made on the presence or absence of alcohol and acid fast bacilli or granulomata. The aspirate was not sent for culture of $M$ tuberculosis.

Anti-tuberculosis treatment was started. The lymphadenopathy progressed despite three months of treatment. Lymph node biopsy was then performed and showed diffuse large B cell lymphoma. Treatment with $\mathrm{CHOP}$ (cyclophosphamide, doxorubicin, vincristine, and prednisolone) and rituximab chemotherapy resulted in a complete remission.

\section{Case 3}

A 16 year old UK-born man of Indian ethnic origin was referred to a respiratory clinic with a nine month history of cervical lymphadenopathy, unproductive cough, sweats, and $4 \mathrm{~kg}$ weight loss. Examination showed hard painless nodes in the right side of the neck but was otherwise normal. Routine blood tests including full blood count were normal. A chest $x$ ray showed multiple bilateral soft tissue shadows with cavitations. As his cough was unproductive there was no sputum to send for further analysis.

Quadruple therapy was started for presumed tuberculosis on clinical grounds alone. After three months his lymphadenopathy had progressed and enlarged inguinal nodes were also present. Lymph node biopsy showed Hodgkin's lymphoma (figs $1 \underline{\underline{\Downarrow}}$ and $2 \underline{\Downarrow}$ ). After an initial remission with ChIVPP/ABVD (chlorambucil, vinblastine, procarbazine, prednisolone/doxorubicin, bleomycin, vinblastine, dacarbazine) he relapsed six months later. He received EPIC (etoposide, prednsiolone, ifosfamide, and cisplatin) salvage chemotherapy and is now in remission after peripheral blood stem cell transplantation. 


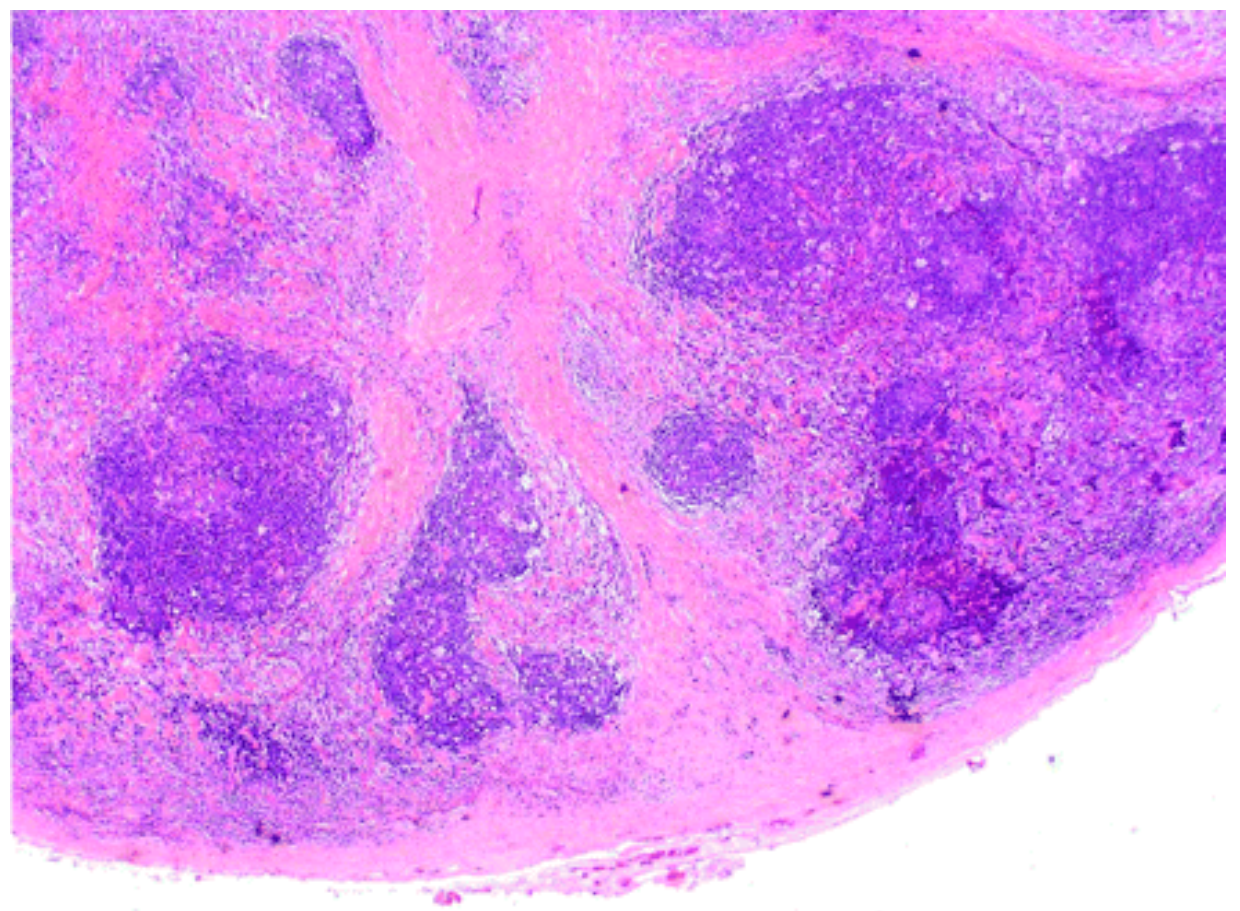

Fig 1 Lymph node biopsy showing features typical of classical nodular sclerosing Hodkin's lymphoma. Collagenous bands of connective tissue (staining pink with eosin) separate areas of abnormal Hodgkin tissue. (2.5x magnification)

$\begin{array}{ll}- & \frac{\text { Download figure }}{\text { Open in new tab }} \\ \text { Download powerpoint }\end{array}$

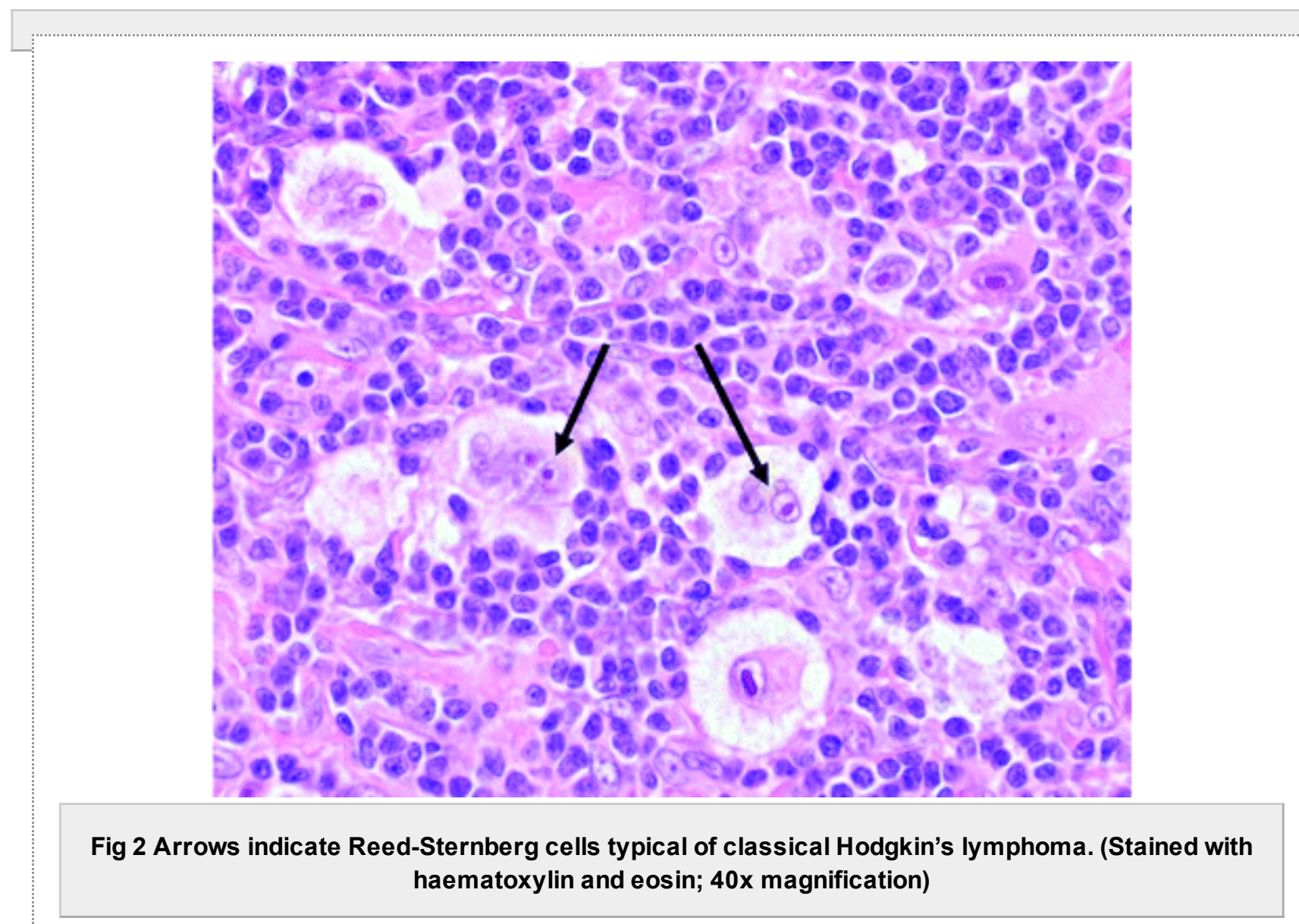




\section{Discussion}

Fine needle aspiration is a rapid, relatively cheap, and minimally invasive procedure. Fine needle aspirate cytology in the diagnosis of lymphoma has its advocates, particularly when combined with immunophenotyping and genetic analysis. The British Committee for Standards in Haematology, however, recommends lymph node biopsy to establish a primary diagnosis of lymphoma whenever possible. 1 In some centres a core biopsy, with or without immunophenotyping or genetic analysis, will be performed in the first instance, but we prefer to proceed to an excision biopsy.

Lymph node tuberculosis can be confidently diagnosed from fine needle aspiration. The gold standard is culture of Mycobacterium tuberculosis from aspirate, which also allows sensitivity testing but can take several weeks. Rapid diagnosis can be made through microscopy and cytological examination of the aspirate if alcohol and acid fast bacilli are seen, but lymph node tuberculosis is often paucibacillary with negative microscopy and indeed culture, especially in immunocompetent patients. 2 Other features that can be seen on cytological examination and are sufficiently characteristic of tuberculosis to allow treatment to be started are stated in the current NICE guidelines as being the presence of caseating granulomas or granulomas with Langerhan's giant cells. 2

Studies performed in resource poor countries, often in settings where HIV infection is prevalent, have sometimes considered caseation without granuloma and non-caseating granuloma as indicating tuberculosis. 3 However, NICE guidelines for the clinical management and diagnosis of tuberculosis state that the presence of non-caseating granuloma on lymph node aspirate is not sufficient for diagnosis, and that other tests confirming tuberculosis are required.2 This is because non-caseating granulomas are seen in a variety of processes, including malignancies such as lymphoma.4 Although it is not explicitly advocated in NICE guidelines, some tuberculosis specialists consider that, in non-white patients in the UK, aspirating pus from a "cold" lymph node is sufficient to start antituberculosis treatment while waiting for culture results. Given the high rate of positive cultures in non-white patients, we advocate reassessment of the lymphadenopathy if the culture result comes back negative.

Even in countries where tuberculosis is endemic, only a minority of lymphadenopathy is due to tuberculosis, so the World Health Organization recommends that the diagnosis and management of suspected lymph node tuberculosis should include a formal excision biopsy for histology if aspirates for cytology and alcohol and acid fast bacilli do not lead to a definitive diagnosis. 5 The current NICE guidelines, however, state that "persistent lymphadenopathy of over 4 weeks duration in people other than white UK-born should be regarded as TB until proven otherwise." Although the guidelines correctly draw attention to the unusually high risk of tuberculosis in non-white people born outside the UK, we believe that our three patients exemplify the danger of undue emphasis on tuberculosis as a cause of lymphadenopathy, potentially resulting in a decision to start tuberculosis treatment before a firm diagnosis is reached. This danger might be avoided by clearer recommendations for the diagnostic work-up in patients who do not have any of the diagnostic cytological features of tuberculosis (alcohol and acid fast bacilli, caseating granulomas, or granulomas with Langerhans giant cells). Although the NICE guidelines state that conditions other than tuberculosis can cause non-caseating granulomas, the discussion then considers the role of tests for tuberculosis infection (tuberculin test or interferon-gamma test) without 
infection and disease, and so can neither confidently confirm nor confidently exclude tuberculosis as a cause of lymphadenopathy, especially in people born outside the United Kingdom or immunocompromised patients. Our third case emphasises the fact that clinical symptoms (painless lymphadenopathy, sweats, and weight loss), even when cavitations are shown on chest $x$ ray, do not obviate the need to use appropriate pathology specimens to establish a diagnosis.

In all but the most exceptional cases, patients being investigated for lymphadenopathy in the UK should be referred for formal biopsy if their fine needle aspirate is non-diagnostic. This should include a specimen for microbiology which has not been fixed in formalin. Formal biopsy ensures that adequate tissue is obtained to allow confirmation of the diagnosis of tuberculosis in almost all cases where this is the cause; increases the likelihood of obtaining a positive culture for drug sensitivity testing; minimises the risk of alternative diagnoses such as lymphoma being missed or delayed; and identifies rare cases of lymphoma and coexistent tuberculosis. Even when a firm diagnosis of tuberculosis has been made from fine needle aspirate, failure to respond to anti-tuberculosis treatment should prompt timely reassessment for dual pathology or drug resistant disease. As our three cases illustrate, maintaining a low threshold for timely reassessment is even more essential when tuberculosis treatment has been started on less definitive grounds.

\section{Notes}

Cite this as: BMJ 2010;340:c63

\section{Footnotes}

- We thank Peter Isaacson and Sue Goddard for preparing the histology images.

- Contributors: ELC, KMA, and SD'A had the original idea for the article; RSS, KMA, and ECL performed the literature searches; KMA, SD'A, and DCL identified suitable cases; RSS, ELC, DCL, and KMA wrote the article. KMA is guarantor.

- Funding: This work was supported by the UCL/UCLH comprehensive biomedical research centre. ELC is supported by a Wellcome Trust Senior Fellowship in Clinical Tropical Medicine.

- Competing interests: None declared.

- Provenance and peer review: Not commissioned; externally peer reviewed.

- Patient consent obtained.

\section{References}

1. $\downarrow$ British Committee for Standards in Haematology, Royal College of Pathologists. Best practice in lymphoma diagnosis and reporting. January 2010. www.bcshguidelines.com/pdf/best practice lymphoma diagnosis.pdf.

2. National Collaboratina Centre for Chronic Conditions. Tuberculosis: clinical diaanosis and manaaement of 
tuberculosis, and measures for its presentation and control. Royal College of Physicians, 2006. www.nice.org.uk/nicemedia/pdf/CG033FullGuideline.pdf.

3. $\downarrow$ Patil PS, Bern C. Wide needle aspiration cytology in the diagnosis of lymphadenopathy in Zambia. J Clin Pathol 1993;46:806-9. Abstract/FREE Full Text

4. $\downarrow$ Khurana KK, Stanley MW, Powers CN, Pitman MB. Aspiration cytology of malignant neoplasms associated with granulomas and granuloma-like features: diagnostic dilemmas. Cancer1998;84:84-91. CrossRef Medline Web of Science

5. $\downarrow$ World Health Organization. Improving the diagnosis and treatment of smear-negative pulmonary and extrapulmonary tuberculosis among adults and adolescents. Recommendations for HIV-prevalent and resourceconstrained settings. 2007. http://whqlibdoc. who.int/hq/2007/WHO HTM TB 2007.379 eng.pdf.

\section{$\checkmark$ Tweet}

$f$ Like 4

- $\mathbf{G}+1<0$

\section{Article tools}

\section{responses}

- Eespond to this article

- BPrint

- Alerts \& updates

\section{Article alerts}

Please note: your email address is provided to the journal, which may use this information for marketing purposes.

\section{Log in or register:}

Username *
Password *

Log in

$\underline{\text { Register for alerts }}$

(1) If you have registered for alerts, you should use your registered email address as your username - OCitation tools

\section{Download this article to citation manager}

Sellar Rob S, Corbett E L, D'Sa Shirley, Linch David C, Ardeshna Kirit M. Treatment for lymph node tuberculosis BMJ 2010; 340 :c63

- BibTeX (win \& mac)

Download

- EndNote (tagged)

Download

- EndNote 8 (xml)

Download

- RefWorks Tagged (win \& mac)

Download 